\title{
Strategies for altering population intakes of fats and fatty acids
}

\author{
M. J. Gibney \\ Institute of European Food Studies, Trinity College, Dublin 2, Republic of Ireland
}

\begin{abstract}
Quantitative dietary guidelines for fats were first issued in 1977 in the USA and these guidelines have changed little since then. In the UK only $14 \%$ of the population achieve the dietary goal for fat (33\% energy) and only $3 \%$ achieve the target (10\% energy) from saturated fatty acids. Analysis of the Dietary and Nutritional Survey of British Adults reveals that across quartiles of decreasing total fat intake, the actual fatty acid composition of this fat does not alter; i.e. when total fat is lowered, all fatty acid categories are equally lowered. This arises because $85 \%$ of total fat and of each of the categories of fatty acids are provided by just five foods (milk, meat, cereals, spreads and vegetables). When total fat in the UK is lowered, the intake of polyunsaturated fatty acids is lowered. The problem is that if the intake of polyunsaturated fatty acids falls below a threshold of about $5 \%$ energy, the cholesterol-raising properties of certain saturated fatty acids, e.g. myristic acid, are greatly augmented. In order to alter the balance of dietary fatty acids, more data are needed on food choices of those in the population achieving particular targets. These targets cannot be based on existing dietary goals, since so few people attain them. A new set of 'interim attainable dietary guidelines', based on prevailing dietary intakes, must be the basis for establishing sensible food-based dietary guidelines.
\end{abstract}

Cardiovascular disease: Dietary fatty acids: Lipoprotein-cholesterol: Dietary fat guidelines

The purpose of the present paper is to examine the public health strategies for modifying the fatty acid compositions of the diets of populations. There is at present compelling evidence that the composition and amount of dietary fat can significantly influence population risk of diseases such as CHD, cerebrovascular disease and dementia. The present review will focus on cardiovascular disease, and specifically on dietary fatty acids and lipoprotein-cholesterol and triacylglycerol levels. Thus, it will exclude the role of dietary fatty acids in several metabolic systems which may be of significance in cardiovascular disease such as the inflammatory and haemostatic systems.

Shifting population intakes of fatty acids necessarily requires targets, and Kritchevsky (1998) has reviewed the evolution of dietary guidelines in the USA. The earliest quantitative dietary guidelines on dietary fat were published in 1977 (US Senate Select Committee on Nutrition and Human Needs, 1977). This report recommended that total dietary fat should be reduced to $30 \%$ total energy, and that it should be evenly distributed at $10 \%$ total energy from each of saturated fatty acids (SFA), monounsaturated fatty acids and polyunsaturated fatty acids (PUFA). These quantitative dietary guidelines for fat have changed little since then. However, there are good reasons why these ideal goals should be divorced from a possible new set of dietary goals or guidelines which might be characterized by being both 'interim' and 'attainable' (interim attainable dietary guidelines; IADG).

To begin with, the success to date in shifting the balance of dietary fatty acids is limited. In the UK, only $14 \%$ of the population attain the existing dietary goal of $33 \%$ energy from fat, while only $3 \%$ achieve the SFA target of $10 \%$ energy from such fatty acids (Ministry of Agriculture, Fisheries and Food, 1994). Similar findings have been reported for The Netherlands (Hulshof et al. 1993). The problem is in fact more serious than these values would suggest. Table 1 presents data obtained from an analysis of the database of the Dietary and Nutritional Survey of British Adults (Gregory et al. 1990). Intakes of total fat expressed as \% energy are divided into quartiles, and the mean intakes of fatty acid categories are given both as \% energy and as \% dietary fat. Clearly, as total fat intake falls, the intakes of all categories of fatty acids also fall. When the intakes of fatty acid categories are expressed as \% total fat, the compositions of dietary fats across quartiles remains constant. In other words, under free-living conditions the UK population 
Table 1. Patterns of intake of saturated (SFA), monounsaturated (MUFA) and polyunsaturated (PUFA) fatty acids in British subjects ( $n$ 2200) classified according to quartiles of total fat intake ${ }^{\star}$ (Data from Gregory et al. 1990)

\begin{tabular}{|c|c|c|c|c|}
\hline $\begin{array}{l}\text { Quartile ... } \\
\text { Total fat intake (\% energy) }\end{array}$ & $\begin{array}{c}1 \\
34 \cdot 1\end{array}$ & $\begin{array}{c}2 \\
40 \cdot 2\end{array}$ & $\begin{array}{c}3 \\
43.5\end{array}$ & $\begin{array}{c}4 \\
48 \cdot 3\end{array}$ \\
\hline \multicolumn{5}{|l|}{ Percentage energy from: } \\
\hline SFA & 13.8 & 16.7 & 18.2 & $20 \cdot 3$ \\
\hline MUFA & 10.4 & 12.1 & $13 \cdot 2$ & $14 \cdot 8$ \\
\hline PUFA & 4.8 & 5.4 & 5.6 & $6 \cdot 2$ \\
\hline \multicolumn{5}{|c|}{$\begin{array}{l}\text { Percentage composition of dietary } \\
\text { fat from: }\end{array}$} \\
\hline SFA & 44 & 44 & 45 & 45 \\
\hline MUFA & 33 & 33 & 33 & 33 \\
\hline PUFA & 15 & 15 & 14 & 14 \\
\hline
\end{tabular}

- Data exclude trans unsaturated fatty acids and $n-3$ PUFA.

Table 2. Intakes ( $g / d$ ) of saturated (SFA), monounsaturated (MUFA) and polyunsaturated (PUFA) fatty acids from the top five sources of SFA in the British diet ( $n$ 2200) (Data from Gregory et al. 1990)

\begin{tabular}{lcccc}
\hline & SFA & MUFA & PUFA & Total fat \\
\hline Milk and milk products & 8.5 & 3.3 & 0.3 & 13.4 \\
Meat and meat products & 8.3 & 8.3 & 2.0 & 21.5 \\
Cereals and cereal products & 6.6 & 4.7 & 2.6 & 16.9 \\
Fat spreads & 6.2 & 3.0 & 2.4 & 14.1 \\
Vegetables & 2.4 & 3.2 & 2.8 & 9.7 \\
All foods & 37 & 27 & 12 & 88 \\
Percentage from the five foods & 88 & 84 & 85 & 86 \\
\hline
\end{tabular}

lowers all fatty acids equally as it lowers total fat intake. This is not what was intended. The intention was that there would be a disproportionate reduction in SFA, with PUFA and monounsaturated fatty acids at least remaining unchanged, if not shifting slightly in balance. There are important public health nutrition consequences of this pattern of lowering all fatty acid categories equally, which will be discussed later, but it is instructive to begin to explore why this pattern occurs.

Table 2 contains data from Gregory et al. (1990) which examine the intake $(\mathrm{g} / \mathrm{d})$ of fatty acid categories from the five main sources of SFA, ranked according to the level of contribution of each source of SFA intake. It is notable that these five food categories contribute on average $85 \%$ of the intakes of total fat and of each of the three main categories of fatty acids. Individuals who therefore opt to reduce their intakes of these foods will, on average, reduce intakes of all categories of fatty acids equally. The only way this can be prevented would be to change the fatty acid compositions of the fats within these food categories. This might mean moving away from low-fat varieties to full-fat varieties where the fatty acid composition is altered. There are two possible problems with that. One is the public obsession with low-fat products, and the second is the technological problems of processing certain types of foods which necessarily require fats with a high melting point.

With regard to the public obsession with lower-fat products, it would appear that we are entering a new era which will see a debate on the question of whether we should maintain fat levels as they presently are but reduce specifically the intakes of SFA, or whether we should reduce total fat intake, with a disproportionate reduction in SFA. The case for lowering total fat intake inevitably hinges on the proposed role of high-fat diets in predisposing to obesity (Connor \& Connor, 1997). The case against this and for keeping total fat intake constant with a reduction in SFA challenges the evidence that higher-fat diets lead to weight gain, but more importantly shows that low-fat diets raise plasma triacylglycerols and lower plasma HDL-cholesterol, the combined effects of which are deemed to be deleterious (Katan et al. 1997). With regard to the latter argument, lowfat diets do not raise plasma triacylglycerols when modest doses $(1 \mathrm{~g} / \mathrm{d})$ of $n-3$ PUFA are present in the diet (Roche \& Gibney, 1996). Moreover, it is well established that physical activity significantly raises HDL-cholesterol levels in plasma (Hardiman \& Herd, 1988). Thus, the possible deleterious effects of low-fat diets are not evident when the full spectrum of public health nutrition advice applies, which includes adequate intakes of $n-3$ PUFA and adequate levels of physical activity. However, the latter two features are certainly not characteristic of existing lifestyles in developed economies and, therefore, the debate is perfectly justifiable.

At this juncture, it is worth returning to the data in Table 1 , because they may be relevant to this debate. The ability of individual SFA to influence plasma cholesterol varies with chain length. Palmitic acid (16:0) and myristic acid (14:0) have been identified as being the most hypercholesterolaemic (Katan et al. 1994). However, Hayes et al. (1997) have examined the relationship between the most hypercholesterolaemic of the SFA (myristic acid) and the most hypocholesterolaemic PUFA (linoleic acid 18:2n-6) in a meta-analysis of studies in monkeys and human subjects. In the case of the latter, $85 \%$ of the variation in plasma cholesterol (dependent variable) could be explained by the intake of myristic acid and linoleic acid. These authors go on to show that the cholesterol-raising properties of myristic acid are greatly influenced by the dietary intake of linoleic acid. Above a threshold of 5-9 \% energy from linoleic acid, increasing intakes of myristic acid have very modest effects on blood cholesterol. Below this threshold of linoleic acid intake myristic acid has a very potent effect on blood cholesterol. Thus, as free-living UK subjects opt to lower total fat intake, they tend to bring their intake of linoleic acid, the most abundant $n$ - 6 PUFA in the diet, down below the critical $5 \%$ threshold for linoleic acid intake. As they go below this threshold in pursuit of low-fat diets, they may reach a point where myristic acid, even if its intake is lowered, can begin to exert a more significant cholesterolraising effect, thus possibly, in respect of blood cholesterol, partially defeating the purpose of the exercise. These points are being raised only to stimulate debate on the wisdom of what is actually happening in practice, i.e. a reduction in total fat intake with absolutely no change in fatty acid composition.

If, as existing dietary guidelines indicate, the objective is to alter fatty acid compositions of the diets of populations, a renewed strategy in public health nutrition is needed. To begin with, since prevailing population intakes of SFA are so wide of the target set within existing dietary guidelines, the establishment of food-based dietary guidelines cannot 
be based on so small a proportion of the population. It is possible that a new set of guidelines should be established, not necessarily to replace but to complement the existing ones, which might be termed IADG. These guidelines would be based on prevailing intakes of nutrients. The approach to setting IADG could be flexible and pragmatic. This could include an arbitrary target representing that attained by the best tertile of intakes, or it could be one standard deviation above or below the mean intake depending on the desired direction of change. Equally it could be based on what has been achieved in a comparable country over a period of time. The key value of IADG is that they now allow for the construction of appropriate food-based dietary guidelines, as advocated by the UN agencies (World Health Organization, 1996). Such guidelines would examine the patterns of food intake of those achieving the IADG and seek to move the total population in that direction. For example, and ignoring present dietary guidelines, one could compare the patterns of food intake of those who simultaneously achieve certain IADG e.g. $36 \%$ fat energy, $14 \%$ SFA energy and $6 \%$ PUFA energy, against those who achieve less-desirable combinations. With respect to a given food, this will help determine which of the following options are the most likely to succeed: changing the percentage of consumers of the food, changing the frequency of consumption, changing the serving size, or changing the composition of the fat within a food. At present our strategy is based on highly simplistic assumptions within a highly complex web of food-food, food-nutrient and nutrient-nutrient interdependencies, and an equally complex web of meal-foods interactions.

It is clear that present strategies for modifying population fatty acid intakes are not as successful as public health nutrition would like them to be. One possible future direction might be the application of biotechnology to modify fatty acid compositions of different oils such that they satisfy both nutritional and processing needs. Equally, new means of incorporating oils into foods to isolate them during processing may assist in this regard.

\section{References}

Connor WE \& Connor SL (1997) Should a low-fat, highcarbohydrate diet be recommended for everyone. The case for a low-fat, high-carbohydrate diet. New England Journal of Medicine 337, 562-563.

Gregory J, Foster K, Tyler H \& Wiseman M (1990) The Dietary and Nutritional Survey of British Adults. London: H. M. Stationery Office.

Hardiman A \& Herd SL (1988) Exercise and postprandial lipid metabolism. Proceedings of the Nutrition Society 57, 63-72.

Hayes KC, Kohsla P, Hajri T \& Pronczuk A (1997) Saturated fatty acids and LDL receptor modulation in humans and monkeys. Prostaglandins, Leukotrienes and Essential Fatty Acids 57, 411418.

Hulshof KF, Lowik MR, Kistemaker C. Hermus RJ, ten Hoor F \& Ockhuizen T (1993) Comparison of dietary intake data with guidelines: some potential pitfalls (Dutch Nutrition Surveillance System). Joumal of the American College of Nutrition 12, 176185.

Katan MB, Grundy SM \& Willett WC (1997) Should a low-fat, high-carbohydrate diet be recommended for everyone. Beyond low-fat diets. New England Journal of Medicine 337, 562-566.

Katan MB, Zock PL \& Mensink RP (1994) Effects of fats and fatty acids on blood lipids in humans: an overview. American Journal of Clinical Nutrition 60, 1017S-1022S.

Kritchevsky D (1998) History of recommendations to the public about dietary fat. Journal of Nutrition 128, 449S-452S.

Ministry of Agriculture, Fisheries and Food (1994) The Dietary and Nutritional Survey of British Adults. A Future Analysis. London: H. M. Stationery Office.

Roche HM \& Gibney MJ (1996) Postprandial triacylglycerolaemia: the effect of low-fat dietary treatment with and without fish oil supplementation. European Journal of Clinical Nutrition 50, $617-624$

US Senate Select Committee on Nutrition and Human Needs (1977) Dietary Goals for the United States. Washington, DC: US Government Printing Office.

World Health Organization (1996) Preparation and Use of Foodbased Dietary Guidelines. Report of a Joint FAO/WHO Consultation. Geneva: WHO. 